Polymer Journal, Vol. 8, No. 5, pp 457-465 (1976)

\title{
A Carbon-13 NMR Study of Molecular Motion of Polystyrene in Solution
}

\author{
Yoshio InOue and Tetsuo Konno \\ Department of Polymer Chemistry, Tokyo Institute of Technology, \\ Ookayama, Meguro-ku, Tokyo, Japan.
}

(Received May 17, 1976)

\begin{abstract}
The molecular motion of polystyrene in solution has been investigated quantitatively using carbon-13 NMR. It is concluded that the motion can be characterized by three contributions, namely, segmental motion and two kinds of internal rotation of the phenyl ring, and that the phenyl ring is rotating more freely above $50^{\circ} \mathrm{C}$. Free rotation of the phenyl ring is hindered by a energy barrier, which is probably due to a steric hindrance between the hydrogens in the phenyl ring and the methine hydrogen in the adjacent monomer unit. This model is consistent with the fact that a transition of polystyrene at about $50^{\circ} \mathrm{C}$ occurs both in solutions and in bulk.

KEY WORDS Polystyrene / ${ }^{13} \mathrm{C}$ NMR / Molecular Motion / Internal Rotation / Transition / Spin-Lattice Relaxation /
\end{abstract}

It has been generally accepted that solutions of isotactic and atactic polystyrene show an anomalous temperature dependence in solution properties at about 50 and $80^{\circ} \mathrm{C}^{1-5}$ A transition was observed at about $80^{\circ} \mathrm{C}$ for isotactic and atactic polystyrene in several solvents in the temperature dependence of the second virial coefficient, in the mean radius of gyration, and in other properties. This transition is firstorder, ${ }^{6-8}$ and Reiss ${ }^{6,7}$ has proposed that it arises from the transition between a $3_{1}$ helix and a random coil in the isotactic sequences of both polymers.

On the other hand a transition at about $50^{\circ} \mathrm{C}$ in isotactic and atactic polystyrene both in solution and in bulk has been reported. ${ }^{1}$ As $50^{\circ} \mathrm{C}$ is well below the glass-transition temperature (ca. $105^{\circ} \mathrm{C}$ ), it was thought that the major molecular process can not be a conformational change but an intramolecular change of more local molecular motions. It was proposed that rotation of the phenyl ring may be hindered by the $\alpha$ and $\beta$ main-chain hydrogens, and that the transition at about $50^{\circ} \mathrm{C}$ may be due to a sudden change in the mobility of the phenyl ring. ${ }^{9}$ However, the molecular motion has not been heretofore studied quantitatively; we have used ${ }^{13} \mathrm{C}$ NMR to elucidate the molecular process occurring in this transition.
Although physical properties of polymer solutions have been studied with proton NMR, data are often rather complex to analyze because of a narrow range of chemical shifts (ca. $10 \mathrm{ppm}$ ) and of splitting of peaks due to spin-spin coupling. Proton-decoupled natural abundance ${ }^{13} \mathrm{C}$ NMR spectra are easier to analyze because of a wide range of chemical shifts (ca. $250 \mathrm{ppm}$ ) and the absence of complex spin-spin coupling. Partially relaxed Fourier transformed (PRFT) spectra permit measurement of the ${ }^{13} \mathrm{C}$ spinlattice relaxation times for all resolved peaks of complex molecules. It is of interest to measure the ${ }^{13} \mathrm{C}$ spin-lattice relaxation times of individual carbons in molecules, as the molecular motion of individual groups can be estimated quantitatively. Proton magnetic relaxation times are difficult to analyze quantitatively in terms of molecular motion because of the difficulty of separating intramolecular and intermolecular contributions. The latter contribution to ${ }^{13} \mathrm{C}$ spin-lattice relaxation is generally negligible. ${ }^{10}$

Furthermore, carbon-13 spin-lattice relaxation times of protonated carbons in large molecules may be analyzed simply if relaxation is overwhelmingly dominated by dipolar interactions with directly bonded protons. ${ }^{11}$ In polystyrene with molecular weight about $10^{4}$ or higher, spin- 
lattice relaxation times are independent of molecular weight within experimental error. ${ }^{11}$ This reflects effective rotational correlation times determined by the rate of segmental motion rather than by overall reorientation of the molecules. ${ }^{11,12}$

\section{EXPERIMENTAL}

\section{Materials}

Polystyrene $^{13}\left(M_{n}=275,000, M_{w}=380,000\right)$ was prepared with $\mathrm{Al}\left(\mathrm{C}_{2} \mathrm{H}_{5}\right)_{3} / \mathrm{TiCl}_{3}$ catalyst. The component insoluble in methyl ethyl ketone was used as the isotactic sample. Atactic polystyrene $\left(M_{n}=180,000, M_{w}=370,000\right)$ was a standard sample (no. 706) from the National Bureau of Standards (U.S.A.).

\section{Methods}

Carbon-13 NMR spectra were measured using a JEOL JNM PS-100 spectrometer equipped with the PFT-100 Fourier transform system operating at $25.15 \mathrm{MHz}$, and a magnetic drum with $8 \mathrm{~K}$ words ( 1 word $=16$ bits) as an auxiliary memory device, yielding 4096 data points in the Fouriertransformed spectrum. The $90^{\circ}$ pulse width was about $20 \mu \mathrm{sec}$. The apparatus was equipped with an internal ${ }^{2} \mathrm{D}$ lock and noise modulated proton decoupler. The $90^{\circ}$ pulse recycle times were chosen to be at least five times the longest spin-lattice relaxation time of the polymer. Samples were contained in $8 \mathrm{~mm}$ O.D. glass tubes and were not degassed since the relaxation times of the polymer are not substantially affected by the presence of dissolved oxygen, at least at atmospheric pressure. ${ }^{14}$ The solvents used were toluene- $d_{8}$ and cyclohexane- $d_{12}$, both obtained from E. Merck A. G. The polymer concentration was $15 \%(\mathrm{wt} / \mathrm{vol})$. Measurements were made at $25^{\circ} \mathrm{C}$ to $90^{\circ} \mathrm{C}$.

In Figure 1 are shown carbon-13 NMR spectra of isotactic and atactic samples in toluene- $d_{8}$. Assignment of individual peaks to corresponding carbons in the polymer was carried out by comparison with the carbon-13 spectrum of ethylbenzene. ${ }^{15}$ The solvent peaks are greatly attenuated and pratically negligible compared to the polymer peaks, because the repetition times of the $90^{\circ}$ pulses are much less than the spin-lattice relaxation times of the carbons in the solvent and because of splitting due to ${ }^{13} \mathrm{C}-{ }^{2} \mathrm{D}$ spin coupling.

\section{NOE Measurement}

The nuclear Overhauser effect for the methylene and methine carbons of the isotactic poly-
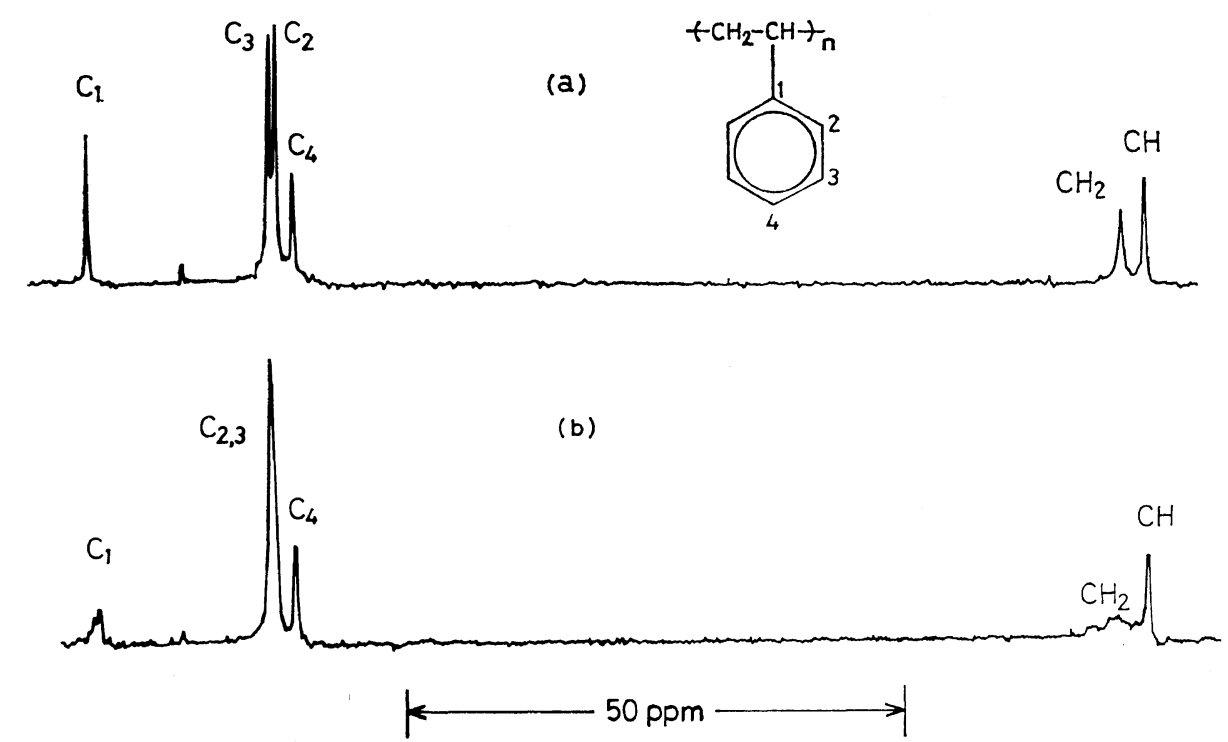

Figure 1. Proton-decoupled, natural abundance ${ }^{13} \mathrm{C}$ FT NMR spectra of (a) isotactic and (b) atactic polystyrene in toluene- $d_{8}$ at $25.15 \mathrm{MHz}$ and $25^{\circ} \mathrm{C}$. The spectra are from 512 accumulations of time-domain signals with a repetition time of $1.2 \mathrm{sec}$. 
mer was estimated directly by comparing the integral intensities of proton-decoupled and proton-coupled resonances. The nuclear Overhauser enhancement (NOE) for aromatic carbons was obtained from comparison of intensities of proton decoupled aromatic resonances with those of methine and methylene resonances.

\section{$T_{1}$ Measurements}

Spin-lattice relaxation times $\left(T_{1}\right)$ for each carbon were determined from proton decoupled PRFT spectra,${ }^{16}$ obtained by means of the conventional $180^{\circ}-t-90^{\circ}$ pulse sequence, where $t$ is the time interval between 180 and $90^{\circ}$ pulses. The intensity $M(t)$ of peaks obtained from sets of such PRFT spectra was used to determine $T_{1}$ according to the expression,

$$
M(t)=M_{0}\left[1-2 \exp \left(-t / T_{1}\right)\right]
$$

where $M_{0}$ is the equilibilium intensity of the spectrum. The assumption of exponential decay in eq 1 was justified by the good linearity in the plots of $\log \left[M_{0}-M(t)\right] v s . t$. The $T_{1}$ 's were calculated for more six time intervals $(t)$ by the least-squares method. The maximum error was within $\pm 5 \%$. More than two runs were carried out.

\section{RESULTS AND DISCUSSION}

\section{Nuclear Overhauser Effect}

In analyzing carbon-13 spin relaxation, one must consider at least three possible relaxation mechanisms, ${ }^{17}$ namely, chemical-shift anisotropy, spin-rotation and ${ }^{13} \mathrm{C}-{ }^{1} \mathrm{H}$ dipole-dipole interactions, even though the last is expected to dominate for protonated carbons in macromolecules. ${ }^{11}$

Interaction of chemical-shift anisotropy is usually negligible in ${ }^{13} \mathrm{C}$ relaxation even with nonprotonated carbons, and it is only at low temperature and at high magnetic field that this mechanism becomes dominant. Hence we can ignore interaction of chemical-shift anisotropy in our experiments.

If $\omega \tau$ (eq 2,3 ) is less than unity, it is easy to separate the spin-rotation and dipolar mechanisms on the basis of their different temperature dependences: i.e., when the spin-rotation mechanism is predominant, $T_{1}$ becomes shorter and NOE becomes smaller with increasing tem-

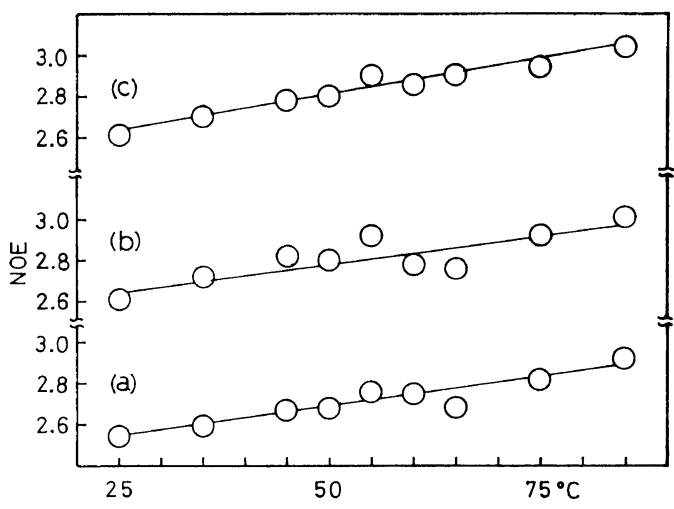

Figure 2. Temperature dependence of NOE's in isotactic polystyrene in toluene- $d_{8}$ : (a) methine and methylene carbons; (b) $\mathrm{C}(4)$; (c) $\mathrm{C}(2)$ and $\mathrm{C}(3)$.

peratures, ${ }^{10,17}$ while $T_{1}$ becomes longer and the NOE becomes larger with increasing temperatures, when ${ }^{13} \mathrm{C}-{ }^{1} \mathrm{H}$ dipole-dipole interaction is predominant. ${ }^{10,17}$ If the dipolar mechanism is predominant and the extreme-narrowing condition is fulfilled, the NOE has a maximum value of 2.988. Figure 2 shows the temperature dependence of the NOE's in the isotactic polystyrene sample. Evidently the NOE increases with increasing temperatures, yielding a maximum NOE at about $80^{\circ} \mathrm{C}$ for the phenyl carbons. As $\omega \tau$ is believed to be smaller than unity in our experiment, this suggest that the major relaxation mechanism is the dipole-dipole interaction. The NOE's are less than the maximum at low temperature. This may be due to the fact that the extreme-narrowing condition is not fulfilled in single correlation time treatment. ${ }^{18}$

It is difficult to study the transition of polystyrene in solution by using the temperature dependence of the NOE's because the uncertainty in NOE values is relatively large (experimental error, $\pm 10 \%$ ) and because the NOE is sensitive to only a limited range of molecular motion. ${ }^{19}$

\section{Linewidth}

Since the peaks for atactic polystyrene are broadened by long accumulation, inhomogenities in the magnetic field, and peak splitting due to tacticity, it is difficult to measure accurate linewidths. Furthermore there are complicated motional effects on $T_{2} \cdot{ }^{16}$ Thus we cannot discuss the segmental motion quantitatively from the linewidth, and can only conclude whether 
a transition is observable at about $50^{\circ} \mathrm{C}$ and whether our result is consistent with the proton NMR measurements.
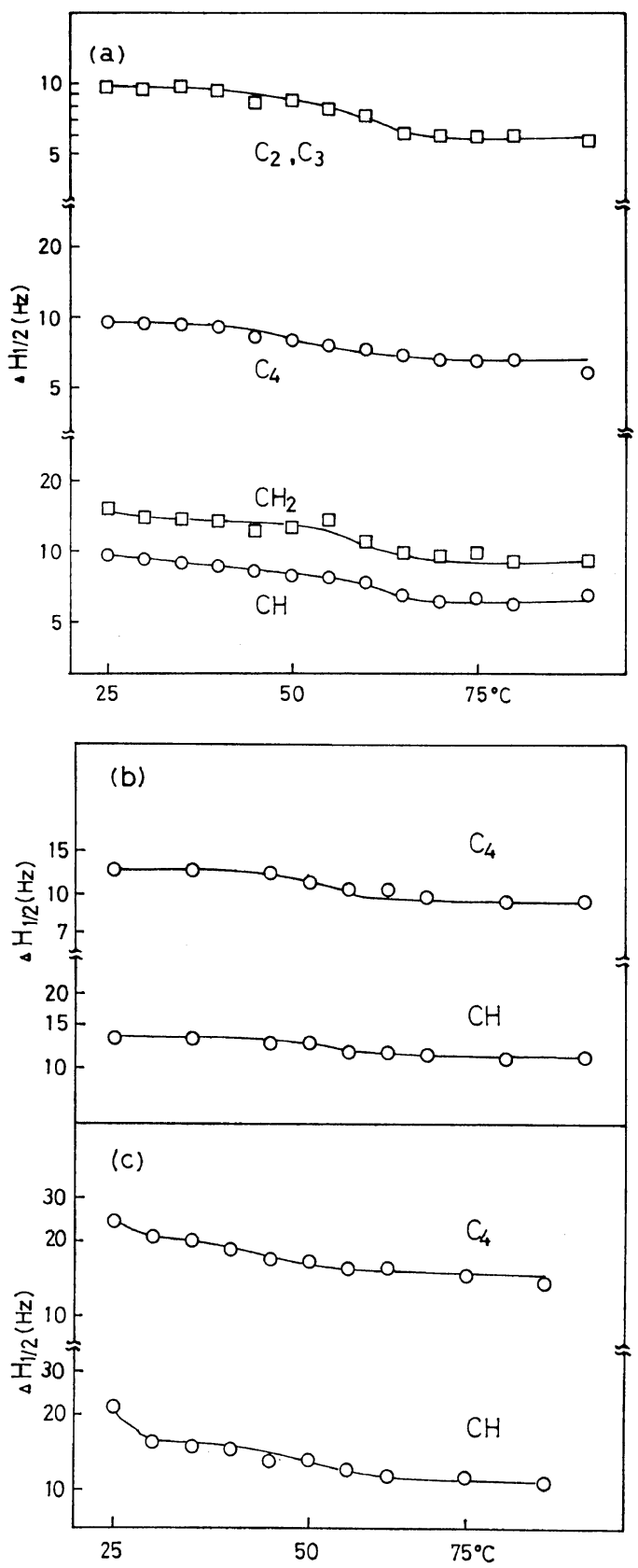

Figure 3. Temperature dependence of linewidth $\left(\Delta H_{1 / 2}\right)$ deduced from normal spectra: (a) isotactic sample in toluene- $d_{8}$; (b) atactic sample in toluene$d_{8}$; (c) atactic sample in cyclohexane- $d_{12}$.
In Figure 3 is shown the temperature dependence of the linewidth $\left(\Delta H_{1 / 2}\right)$ of the carbon-13 resonances of isotactic and atactic samples in solution. In the isotactic sample, linewidths decrease continuously, and the reduction is most rapid between 40 and $60^{\circ} \mathrm{C}$. This behavior coincides quantitatively with results from proton NMR. ${ }^{2,9}$ In the atactic sample in toluene- $d_{8}$ the reductions of linewidth between 40 and $60^{\circ} \mathrm{C}$ are smaller and the linewidths are relatively larger, probably owing to the chemical shift difference between isotactic and syndiotactic sequences. The behavior of atactic polymer in cyclohexane- $d_{12}$ is similar to that in toluene- $d_{8}$, except near $25^{\circ} \mathrm{C}$, but the linewidthes are greater and the reduction in linewidth with increasing temperatures between 40 and $60^{\circ} \mathrm{C}$ is smaller. Since cyclohexane is a poor solvent for atactic polystyrene, this behavior is expected. Atactic polystyrene at this concentration in cyclohenane precipitates at $27^{\circ} \mathrm{C}$, hence the peaks become very broad at $25^{\circ} \mathrm{C}$. Of course isotactic polystyrene does not dissolve in cyclohexane. These results seem to suggest that the transition in polystyrene at about $50^{\circ} \mathrm{C}$ is insensitive to the differences of configuration and the kind of solvent, as previous authors ${ }^{1-3}$ observed.

\section{Molecular Motion Deduced from Spin-Lattice Relaxation Time}

Spin-Lattice Relaxation Time. In Figure 4 is shown the temperature dependence of $T_{1}$ for carbons in both samples in solution. Figure 4 indicates that $T_{1}$ values for the methylene, methine and $\mathrm{C}(4)$ carbons (Descriptions are those in Figure 1) increase linearly with increasing temperatures, while for $\mathrm{C}(2)$ and $\mathrm{C}(3)$, breaks appear at about $55^{\circ} \mathrm{C}$. This behavior is common to the isotactic and atactic samples in toluene$d_{8}$ and the atactic sample in cyclohexane- $d_{12}$. Previously reported experiments ${ }^{1-5}$ also showed a transition at about $50^{\circ} \mathrm{C}$. The mechanism of this transition is discussed further below.

Figure 4 indicates that the $T_{1}$ for methylene carbons is one half that for methine carbons within experimental error, and that the $T_{1}$ values for both samples in toluene- $d_{8}$ are larger than those in cyclohexane- $d_{12}$. This behavior may relate to the fact that toluene is a good solvent and cyclohexane is a poor solvent, and that the 


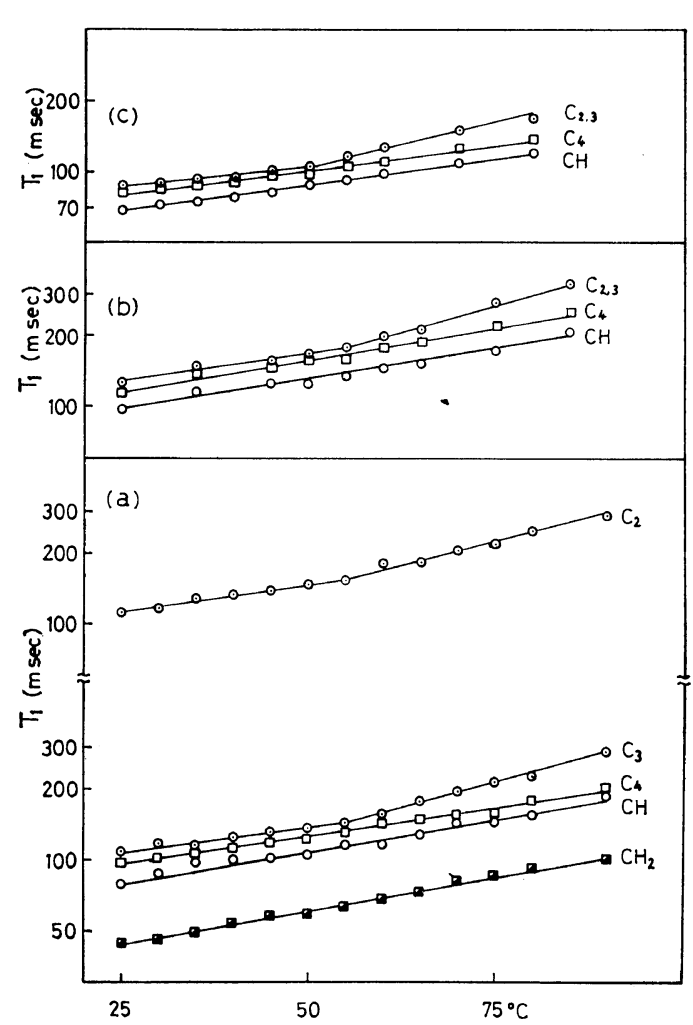

Figure 4. Temperature dependence of the spinlattice relaxation time $\left(T_{1}\right)$ : (a) the isotactic sample in toluene- $d_{8}$; (b) the atactic sample in toluene- $d_{8}$; (c) the atactic sample in cyclohexane- $d_{12}$.

chain segments are more mobile in toluene than in cyclohexane. The $T_{1}$ values are larger for the atactic than for the isotactic sample, and this may relate to the fact that atactic polystyrene is soluble in cyclohexane but isotactic polystyrene is not. The temperature dependences of both $T_{1}$ and linewidth indicate that the transition near $50^{\circ} \mathrm{C}$ occurs irrespective of polymer configuration, conformation, and solvent species.

Correlation Time. If the ${ }^{13} \mathrm{C}-{ }^{1} \mathrm{H}$ dipolar interaction is predominant, and segmental motion is isotropic, $T_{1}$ is given by $^{20}$

$$
\begin{aligned}
\frac{1}{T_{1}}= & \frac{h^{2}}{10} \gamma_{\mathrm{C}}^{2} \gamma_{\mathrm{H}}^{2} \sum_{i} r_{i}^{-6} \\
& \times\left[f\left(\omega_{\mathrm{C}}-\omega_{\mathrm{H}}\right)+3 f\left(\omega_{\mathrm{C}}\right)+6 f\left(\omega_{\mathrm{C}}+\omega_{\mathrm{H}}\right)\right]
\end{aligned}
$$

where

$$
f(\omega)=\tau\left(1+\omega^{2} \tau^{2}\right)^{-1}
$$

$\gamma_{\mathrm{C}}$ and $\gamma_{\mathrm{H}}$ are gyromagnetic ratios for ${ }^{13} \mathrm{C}$ and ${ }^{1} \mathrm{H}, \omega_{\mathrm{C}}$ and $\omega_{\mathrm{H}}$ are the Larmor frequencies for ${ }^{13} \mathrm{C}$ and ${ }^{1} \mathrm{H}$, respectively, $r_{i}$ is the distance between the carbon of interest and the $i$-th proton, and $\tau$ is the single rotational correlation time for the carbon of interest.

If the segmental motion (hereafter, this motion is designated as the $R$ motion) is isotropic in polystyrene, $T_{1}$ for the methylene carbon must be half that for the methine carbon $\left(T_{1}(\mathrm{CH})\right)$ according to eq 2 . The experimental results confirm this prediction, the methylene carbons are in fact one half of those of the methine carbons. Hence the $R$ motion can be expressed by a single correlation time $(\tau(R))$.

When there is an internal rotation, the effective correlation time $(\tau(I))$ is described as the sum of two contributions as follows, ${ }^{21}$

$$
\frac{1}{\tau(I)}=\frac{1}{\tau(R)}+\frac{1}{\tau(G)}
$$

where $\tau(\boldsymbol{G})$ is the correlation time of internal rotation. The quantity $\tau(I)$ cannot be calculated directly using eq 2 , when molecular motion is anisotropic due to the contribution from internal rotation. To obtain the value of $\tau(I)$, the $T_{1}$ ratio $(1 / \chi)$ is defined by, ${ }^{21}$

$$
\frac{1}{\chi}=\frac{T_{1}(I)}{T_{1}(R)}
$$

where $T_{1}(I)$ is the relaxation time when the relaxation is due to internal rotation. The quantity $\chi$ is calculated from, ${ }^{21}$

$$
\begin{aligned}
\chi= & \frac{1}{4}\left(3 \cos ^{2} \theta-1\right)^{2}+\frac{18}{5+\rho} \sin ^{2} \theta \cos ^{2} \theta \\
& +\frac{9}{4} \frac{1}{1+2 \rho} \sin ^{4} \theta
\end{aligned}
$$

where $\theta$ is the angle between the $\mathrm{C}-\mathrm{H}$ vector and the axis of rotation, and

$$
\rho=\frac{\tau(R)}{\tau(I)}
$$

We can divide the internal rotation of polystyrene into an $\alpha$ and $\beta$ motion, respectively, rotation of the phenyl ring around the $\mathrm{C}(1)$ $\mathrm{C}(4)$ axis and precession of the $\mathrm{C}(1)-\mathrm{C}(4)$ axis in a plane perpendicular to the chain backbone. The reason for dividing internal rotation into two parts is as follows: if the molecular motion 


\section{Y. INOUE and T. KONNO}

in polystyrene is described by the $R$ motion and the $\alpha$ motion only, the $T_{1}$ 's for $\mathrm{C}(2)$ and $\mathrm{C}(3)$ becomes larger than $T_{1}$ for $\mathrm{C}(4)$, as is indicated by eq 4-7; and the $T_{1}$ for the methine carbon must equal that for $\mathrm{C}(4) \quad(\chi=1$ when $\theta=0$ from eq 6). Experimental results indicate that $T_{1}$ 's for $\mathrm{C}(2)$ and $\mathrm{C}(3)$ are larger than $T_{1}$ 's for $\mathrm{C}(4)$, while $T_{1}$ 's for $\mathrm{C}(4)$ are clearly larger than those for the methine carbons at all temperatures, even if the difference between factors $\left(\sum_{i} r_{i}^{-6}\right)$ for individual carbons are considered. The difference between $T_{1}$ values for C(4) and methine carbons are not attributable to systematic experimental error. They cannot be due to anisotropic $R$ motion, because this would be inconsistent with the fact that the $T_{1}$ 's for the methylene carbons are one half of those of the methine ones, and with the fact that the differences are larger in the atactic sample than in the isotactic one whose motion may be more anisotropic than that of the atactic one. We do not think that the differences of $T_{1}$ 's for these carbons can be explained by the distribution of correlation times, though the differences of correlation times obtained from $T_{1}, T_{2}$ and NOE can be explained by this way.

Hence we must consider a different kind of internal rotation (other than the $\alpha$ motion) which enlarges the $T_{1}$ for $\mathrm{C}(4)$ compared with that for methine carbon. Though the phenyl ring is rigidly bonded to the chain backbone in polystyrene, ${ }^{22}$ the new kinds of internal rotation inevitably change the bond angle between $\mathrm{C}(1)-$ $\mathrm{C}(4)$ axis and the chain backbone. The $\beta$ motion which is a window-wiper-like one of $\mathrm{C}(1)$ $\mathrm{C}(4)$ axis in a plane perpendicular to the chain backbone. The $\beta$ motion would be expected to make $T_{1}$ for $\mathrm{C}(4)$ larger than that for methine carbon.

As the value of $\theta$ (eq 6) is equal to $90^{\circ}$ for $\mathrm{C}(2,3$, and 4$)-\mathrm{H}$ bond in the case of the $\beta$ motion, the $T_{1}$ 's for $\mathrm{C}(2), \mathrm{C}(3)$ and $\mathrm{C}(4)$ are equally increased compared with that for methine carbon. As the value of $\theta$ is equal to $0^{\circ}$ for $\mathrm{C}(4)-\mathrm{H}$ bond in the case of the $\alpha$ motion, the $T_{1}$ for $\mathrm{C}(4)$ remains constant by the $\alpha$ motion as already described. On the other hand, as the value of $\theta$ is equal to $60^{\circ}$ for $\mathrm{C}(2$ and 3$)-$ $\mathrm{H}$ bond in the case of the $\alpha$ motion, the $T_{1}$ 's for $C(2)$ and $C(3)$ are equally increased. Hence the effective correlation times for individual carbons may be described in terms of the three correlation times for the three elemental motions, as follows.

$$
\begin{aligned}
\frac{1}{\tau(\mathrm{C}(4))} & =\frac{1}{\tau(R)}+\frac{1}{\tau(\beta)} \\
\frac{1}{\tau(\mathrm{C}(2 \text { and } 3))} & =\frac{1}{\tau(R)}+\frac{1}{\tau(\alpha)}+\frac{1}{\tau(\beta)}
\end{aligned}
$$

From eq 5 , we can obtain the $T_{1}$ ratio as follows in the case of $\mathrm{C}(4)$ relaxation,

$$
\frac{1}{\chi}=\frac{T_{1}(\mathrm{C}(4))}{T_{1}(\mathrm{CH})}
$$

and the relation $1 / \chi\left(90^{\circ}\right)-\rho$ gives the value of $\rho$. Then $\tau(\beta)$ is calculated from the values of $\rho$ and $\tau(R)$ as follows.

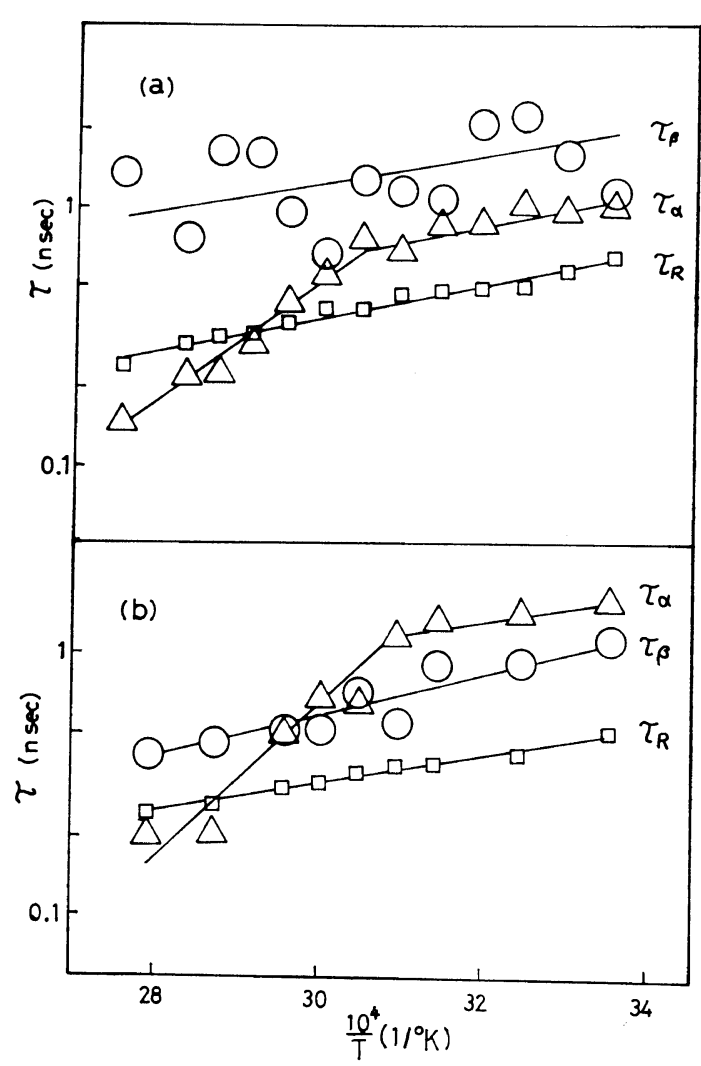

Figure 5. Arrhenius plot of correlation time $(\tau)$ for each molecular motion in polystyrene: (a) the isotactic sample in toluene- $d_{8}$; (b) the atactic sample in toluene- $d_{8}$. 


$$
\tau(\beta)=\frac{\tau(R)}{\rho-1}
$$

In a similar procedure $\tau(\alpha)$ is obtained.

In Figure 5 is the Arrhenius plot of each correlation time of the isotactic and atactic samples in toluene- $d_{8}$. Figure 5 indicates that the Arrhenius plots of the correlation times of the $R$ and $\beta$ motion fall on the respective straight lines, while that of the $\alpha$ motion indicate a break between $50^{\circ}$ and $55^{\circ} \mathrm{C}$. The transition at about $50^{\circ} \mathrm{C}$ must be caused by a modal change in the $\alpha$ motion. In the lower temperature range the $\alpha$ motion of the isotactic sample in toluene- $d_{8}$ is more rapid than $\beta$ motion, while the $\alpha$ motion of the atactic one in toluene- $d_{8}$ is slower than the $\beta$ motion. In the case of the atactic sample in cyclohexane- $d_{12}$ each correlation time at each temperature is similar to that of the atactic one in toluene- $d_{8}$ rather than that of the isotactic one in toluene- $d_{8}$.

The molecular motion was studied by Stockmayer and $\mathrm{Matsuo}^{22}$ for atactic $\operatorname{poly}(m-$ or $p$ halogen styrene) in toluene by measuring the dielectric relaxation at $25^{\circ} \mathrm{C}$. There, the molecular motion was divided into three parts, and each correlation time coincides satisfactory with our corresponding correlation time of the atactic sample in toluene- $d_{8}$ at $25^{\circ} \mathrm{C}$.

Activation Energy. From the temperature dependence of the correlation times we can estimate an activation energy $(\Delta E)$ for the motions responsible for spin-lattice relaxation by use of the relation,

$$
\tau=\tau_{0} \exp (\Delta E / R T)
$$

where $\tau_{0}$ is the correlation time expected at infinite temperature and $R$ is the gas constant. In Table $I$ is presented the activation energies for the three kinds of motion. It is clear that the $\alpha$ motion of the phenyl ring involves surmounting an energy barrier above the transition point, namely, there are two modes of internal rotation designated as the $\alpha$ motion. This energy barrier seems to be independent of configuration and conformation of the sample, since the transition behaviors and the values of the activation energy for the $\alpha$ motion are similar for the isotactic and atactic polymers. The energy barrier for the $\alpha$ motion may be due to a steric
Table I. Activation energy for molecular motions of polystyrene in $15-\%$ (wt/vol) solution in toluene- $d_{8}$ as deduced from Arrhenius plots of the individual correlation times (see Figure 5)

\begin{tabular}{lcccc}
\hline & \multicolumn{4}{c}{$\Delta E(\mathrm{~kJ} / \mathrm{mol})$} \\
\cline { 2 - 5 } Tacticity & $\begin{array}{c}\alpha \text { motion } \\
\text { in the } \\
\text { lower } \\
\text { motion }\end{array}$ & $\begin{array}{c}\alpha \text { motion } \\
\text { in the } \\
\text { tempera- } \\
\text { ture range }\end{array}$ & $\begin{array}{c}\beta \\
\text { tempera- } \\
\text { ture range }\end{array}$ \\
\hline Isotactic & 11.7 & 10.5 & 43.9 & 10.5 \\
Atactic & 10.5 & 10.9 & 56.9 & 15.1 \\
\hline
\end{tabular}

hindrance between hydrogens bonded to $C(2)$ and other hydrogens. As the transition seems to be independent of configuration and conformation of the sample, these other hydrogens may be those on the same or an adjacent monomer unit. Some interatomic distances between specified hydrogens have therefore been calculated to distinguish between these possibilities.

In the calculation of interatomic distances we assume that the bond angles are equal to regular tetrahedral ones in the main chain and to regular hexagonal ones in the phenyl ring, the conformation of isotactic and syndiotactic sequences are $3{ }_{1}$-helix and all trans, ${ }^{23,24}$ respectively, and the bond lengths of the skeletal $\mathrm{C}-\mathrm{C}$ bond, the aromatic $\mathrm{C}-\mathrm{C}$ bond, the aliphatic $\mathrm{C}-\mathrm{H}$ bond, and the aromatic $\mathrm{C}-\mathrm{H}$ bond are $1.53,1.40,1.09$, and $1.08 \AA$, respectively. The calculated minimum interatomic distances between the hydrogens bonded to $\mathrm{C}(2)$ in a rotating phenyl ring and other hydrogens are as follows: the distance from the methine hydrogen in an adjacent monomer unit when the phenyl ring rotates $90^{\circ}$ from the stable position around its $\mathrm{C}_{6}$ axis is $0.72 \AA$, the distance from the hydrogen bonded to $C(2)$ is an adjacent monomer unit of isotactic and atactic sequences are 1.94 and $1.10 \AA$, respectively, and the distances from the methylene and the methine hydrogens in the same monomer unit are 1.58 and $2.47 \AA$, respectively. If $1.2 \AA$ is adopted as the van der Waals radii of hydrogen, ${ }^{25}$ it is concluded that the steric hindrance is mainly caused by the methine hydrogen in an adjacent monomer unit. We can therefore reasonably conclude that rotation of the phenyl ring occurs 
above the transition point, and below this point there is incomplete rotation of the phenyl ring which is confined by the potential-energy barrier causing to the steric hindrance.

\section{Chemical Shift}

Figure 6 shows the temperature dependence of the chemical shifts of each carbon. The chemical shifts are expressed in ppm (parts per million) with respect to the methine carbon peak, negative values corresponding to the lower field. The chemical shifts of the methylene carbons are nearly constant, while those of the phenyl carbons decrease continuously, and the difefences between the shifts at $25^{\circ} \mathrm{C}$ and at $90^{\circ} \mathrm{C}$ are about $0.4 \mathrm{ppm}$.

As the phenyl ring has a magnetic anisotropy due to the ring current, the chemical shift of each carbon may differ depending on whether the phenyl ring rotates or not. The contribution to the chemical shift $(\Delta \sigma)$ from the magnetic anisotropy is calculated by $^{26}$

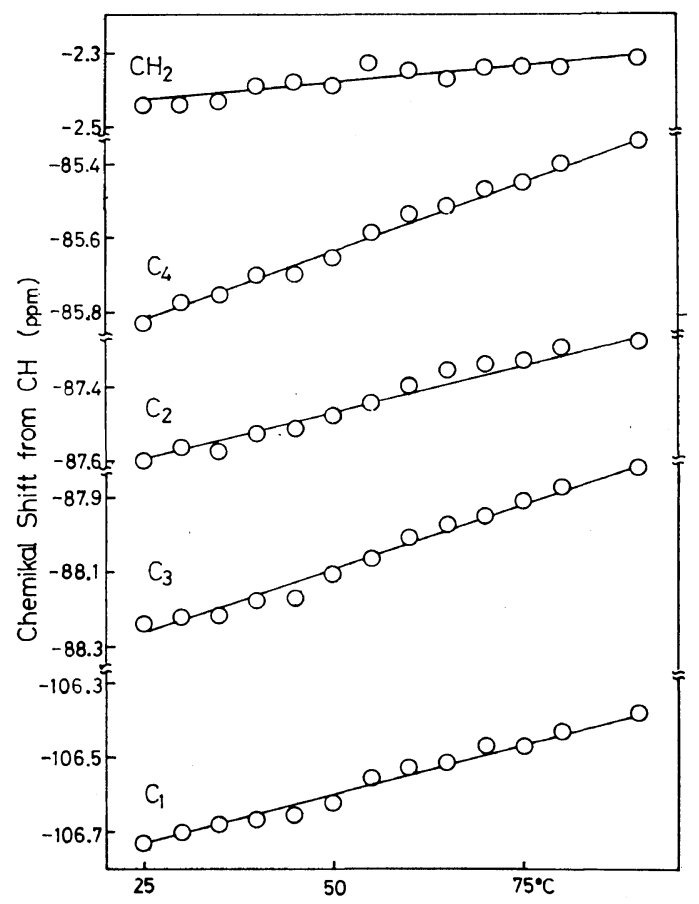

Figure 6. Temperature dependence of the chemical shift for each carbon in isotactic polystyrene in toluene- $d_{8}$. The values of chemical shift are expressed in ppm with respect to the methine carbon peak.

$$
\Delta \sigma=\frac{\Delta \chi}{3 \mathbf{d}^{3}}\left(1-3 \cos ^{2} \phi\right)
$$

where the $\Delta \chi$ is the magnetic anisotropy of the phenyl group and has a value $-90 \times 10^{-30} \mathrm{~cm}^{2}$ esu, $\mathbf{d}$ is the vector from the specified nucleus to the group having the magnetic anisotropy, and $\phi$ is the angle between the direction of the anisotropy axis of the phenyl ring and the internuclear vector $\mathbf{d}$.

The results of calculation show that the values of $\Delta(\Delta \sigma)$ for the skeletal and phenyl carbons are about 0.5 and $0.3 \mathrm{ppm}$, respectively, in the isotactic sequences with $3_{1}$ helical conformation. $\Delta(\Delta \sigma)$ is the difference between $\Delta \sigma$ for the case where the phenyl ring is fixed at the most stable position and $\Delta \sigma$ for the case where it is freely rotating. Thus, the resonance of the skeletal carbons is predicted to approach those of the phenyl carbons by about $0.2 \mathrm{ppm}$ in isotactic polystyrene when the phenyl ring changes from a fixed to a freely rotating state. The calculated value is about one half of the experimental results. This deviation may result from the solvent effect.

\section{CONCLUSION}

From the $T_{1}$ analysis of the polystyrene in solution it is concluded that the transition at about $50^{\circ} \mathrm{C}$ is caused by the modal change of the $\alpha$ motion. The calculation of the interatomic distances suggests that the greatest energy barrier which restricts the free rotation of the phenyl ring is the interaction between the hydrogen bonded to $C(2)$ in the phenyl ring and the methine hydrogen in the adjacent monomer unit. This model coincides with the fact that the transition is not first-order but second-order, that it is observed in bulk, ${ }^{1}$ and that it is independent of the configuration, conformation and species of solvent. In order to confirm that the transition at about $50^{\circ} \mathrm{C}$ is independent of the species of solvent, we chose toluene as a good solvent and cyclohexane as a poor solvent.

It has been reported that the transition of polystyrene in decalin was not observed at about $50^{\circ} \mathrm{C}$ but was observed at about $80^{\circ} \mathrm{C}$ from the temperature dependence of the mean radius of gyration and of the second virial coefficient. ${ }^{7}$ On the other hand it has been reported that 
in decalin the transition at about $50^{\circ} \mathrm{C}$ was observed from the linewidth in proton $\mathrm{NMR}^{2}$ but no transition at about $80^{\circ} \mathrm{C}$. These inconsistencies may be explained in terms of insufficiency of data at about $50^{\circ} \mathrm{C}$ in the first study to conclude whether the transition occur or not, and in terms of ineffectiveness of NMR measurement as a means of observing the change of conformation at about $80^{\circ} \mathrm{C}$. It is therefore of particular interest to note that the intrinsic viscosity has been measured for polystyrene in decalin over the range of $30-100^{\circ} \mathrm{C}$ in steps of about $3^{\circ} \mathrm{C}^{27}$ and two deviations from a smooth curve, one at about $55^{\circ} \mathrm{C}$ and the other at about $80^{\circ} \mathrm{C}$, were found and that a similar result was found from the temperature dependence of the surface tension of solution of atactic polystyrene. ${ }^{28}$ However, the transition at about $80^{\circ} \mathrm{C}$ could not be observed in our results of carbon-13 spin-lattice relaxation measurement of both atactic and isotactic polymers in toluene$d_{8}$. Since the transition at about $80^{\circ} \mathrm{C}$ may be a transition of conformation ${ }^{6}$ with little change in short range motion, NMR is ineffective as a method for study by such a transition, as has already been mentioned. So if the transition at about $80^{\circ} \mathrm{C}$ occurs in toluene, we cannot observe it as effectively as is possible by light scattering or viscometry.

Acknowledgment. The authors thank Professor R. Chûjô for stimulating discussions during this work, and Mr. T. Ikuta for supplying the sample of isotactic polystyrene. This work was partially supported by the Grant-in-Aid for Scientific Reseach from the Ministry of Education, Science, and Culture, Japan (1974).

\section{REFERENCES}

1. G. Moraglio and F. Danusso, Polymer, 4, 445 (1963).

2. Kang-Jen Liu and R. Ullman, ibid., 6, 100 (1965).

3. P. Mason and B. J. Rigly, ibid.; 6, 90 (1965).
4. C. Reiss and H. Benoit, C.R. Acad. Sci., 256, 268 (1961).

5. G. Weill and R. Reeb, ibid., 263, 21 (1966).

6. C. Reiss, J. Chim. Phys., 1299 (1966).

7. C. Reiss, ibid., 1607 (1966).

8. C. Reiss, ibid., 1319 (1966).

9. J. B. Helms and G. Challa, J. Polym. Sci., Part $A-2,10,1447$ (1972).

10. E. von Goldammer, H. D. Lüdermann, and A. Müller, J. Chem. Phys., 60, 4592 (1974).

11. A. Allerhand and R. K. Hailstone, ibid., 56, 3718 (1972).

12. J. E. Anderson, K. J. Liu, and R. Ullman, Disc. Faraday Soc., 49, 257 (1970).

13. Y. Inoue, A. Nishioka, and R. Chûjô, Makromol. Chem., 156, 207 (1972).

14. Y. Inoue, A. Nishioka, and R. Chûjô, J. Polym. Sci. Polym. Ppys. Ed., 11, 2237 (1973).

15. M. Yamazaki, T. Takeuchi, and K. Matsushita, Kogyo Kagaku Zasshi (J. Chem. Soc. Japan, Ind. Chem. Sect.), 74, 656 (1971).

16. A. Abragm, "The Principles of Nuclear Magnetism," Oxford Univ. Press, Mass., 1961.

17. G. C. Levy, J. D. Cargioli, and F. A. L. Anet, J. Amer. Chem. Soc., 95, 1527 (1973).

18. J. Schaefer, Macromolecules, 6, 882 (1973).

19. D. Doddrell, V. Glushko, and A. Allerhand, J. Chem. Phys., 56, 3683 (1972).

20. J. Schaefer and D. F. S. Natusch, Macromolecules, 5, 416 (1972).

21. A. Allerhand, D. Doddrell, and R. Komoroski, J. Chem. Phys., 55, 189 (1971).

22. W. H. Stockmayer and K. Matsuo, Macromolecules, 5, 766 (1972).

23. F. A. Bovey, F. P. Hood, E. W. Anderson, and L. C. Snyder, J. Chem. Phys., 42, 3900 (1965).

24. F. Heatley and F. A. Bovey, Macromolecules, 1, 301 (1968).

25. A. D. Willians and P. J. Flory, J. Amer. Chem. Soc., 91, 3111 (1969).

26. T. Ikuta, I. Ando, and A. Nishioka, Kobunshi Kagaku (Chem. High Polymers), 30, 5 (1973).

27. W. R. Krigbaum, F. Mark, J. G. Pritchard, W. L. Hunter, and A. Cifferri, Makromol. Chem., 65, 101 (1963).

28. A. W. Neum nn, R. J. Good, P. K. Basu, and G. J. Johnston, J. Macromol. Sci., 7, 525 (1973). 